\title{
The effect of acute taurine ingestion on repeat sprint performance
}

\author{
L. Bennis ${ }^{1}$, M. Doyle ${ }^{2}$, K. Fanning ${ }^{2}$ and B.P. Carson ${ }^{2,3}$ \\ ${ }^{1}$ School of Agriculture and Food Science, University College Dublin, Belfield, Dublin 4, Ireland, \\ ${ }^{2}$ Department of Physical Education \& Sports Sciences, Faculty of Education and Health Sciences, University of \\ Limerick, Limerick, Ireland and \\ ${ }^{3}$ Health Research Institute, University of Limerick, Limerick, Ireland
}

Many field based sports include bouts of maximal or sub-maximal sprinting with short recovery periods known as repeated sprint ability (RSA). RSA is defined as sprints short in duration (less than 10 seconds) with brief recovery periods ( $<60$ seconds) usually resulting in a substantial decrease in performance ${ }^{(1)}$. It is an important characteristic of performance in numerous intermittent sports such as soccer, rugby, tennis and GAA. Taurine (TA) is a sulfonic amino acid commonly found in energy drinks. The effect of acute TA supplementation has been investigated in endurance performance. One study has investigated acute TA supplementation in team sports players, demonstrating performance improvements across 3 Wingate tests compared with a placebo (PLA) $)^{(2)}$, however no studies have yet investigated the effect of acute TA supplementation on RSA which is the aim of this study.

A total of 7 young, healthy, recreationally active males and females ( 5 male, 2 female; age $21.3 \pm 1.9$; body mass, $75.4 \pm 10.5 \mathrm{~kg}$ ) were recruited and undertook 3 RSA trials using a $40 \mathrm{~m}$ multiple shuttle test. This incorporated $10 \mathrm{x} 40 \mathrm{~m}$ sprints with two $180^{\circ}$ changes in direction, with $30 \mathrm{~s}$ recovery between each sprint. Sprint times (s) were recorded using photoelectric timing gates (WITTY). Each participant completed a familiarisation session ${ }^{(3)}$, followed by TA $(50 \mathrm{mg} / \mathrm{kg}$ body weight) or PLA trials in a randomised, double blind, crossover design with a washout period of 7 days between each trial. Analysis of sprint performance decrement $\left(\mathrm{S}_{\mathrm{dec}}\right)$, fastest time (FT), slowest time (ST) and mean time (MT) of sprint performance were conducted using SPSS to compare across conditions using a paired samples t-test.

RSA measured by $\mathrm{S}_{\mathrm{dec}}$ did not differ between TA and PLA (TA: $4.4 \pm 2 \%$; PLA: $5.7 \pm 2.4 \% ; p=.301$ ). There was no significant difference observed between TA and PLA trials for FT (TA: $8.68 \pm 0.36 \mathrm{~s}$; PLA: $8.53 \pm 0.45 \mathrm{~s} ; p=.423$ ), ST: (TA: $9.32 \pm 0.43 \mathrm{~s}$; PLA: $9.44 \pm 0.55 \mathrm{~s} ; p=.558)$ or MT: (TA: $9.06 \pm 0.39 \mathrm{~s}$; PLA: $9.02 \pm 0.46 \mathrm{~s} ; p=.731)$.

The findings of this study suggest that an oral acute dose of TA offers no performance advantage in short duration high intensity RSA such as the $40 \mathrm{~m}$ multiple shuttle test when compared to PLA.

1. Girard O, Mendez-Villanueva A \& Bishop D (2011) Sports Med 41, 673-94.

2. Warnock R et al. (2017) IJSPP 12, 1341-1347.

3. Glaister M et al. (2009) JSSM 8, 77-82. 